\title{
Optical characterization for nearly spherical gold colloidals via their polarization response
}

\author{
Bassam Al-Qadi and Toshiharu Saiki \\ School of Integrated Design Engineering, Keio University \\ 3-14-1 Hiyoshi, Kohoku, Yokohama, Kanagawa 233-8522, Japan \\ bassqqad@yahoo.com
}

\begin{abstract}
We optically characterized colloidal gold nanoparticles using polarized light scattering which is utilized to investigate both optical anisotropy and aspect ratio of the particle and to observe particle's rotational dynamics. By monitoring time-trace of the polarized scattering from a particle, we could observe rotational dynamics as fluctuations in the measured anisotropy. With typical particle dimension of $97 \mathrm{~nm}$, the maximum anisotropy was measured experimentally to be 0.1 to 0.5 , and with comparison to our analytical model, this range is equivalent to an aspect ratio range of 1 to 1.3 , which reveals that the colloidals are predominately spherical as predicted by transmission electron microscope (TEM) images analysis. Our method has the advantage to be simple, easy to implement, and can yield access to different projections of the particle due to its rotation. Polarized light scattering can be an ultrasensitive measure for biosensing applications.
\end{abstract}

Keywords: gold nanoparticles, optical anisotropy, polarization, scattering.

\section{INTRODUCTION}

Bioimaging using optical methods forms a major thrust of biophotonics. It is an area of very intense research with a wide range of applications. Optical imaging offer specific advantages including ease of implementation, high spatial and temporal resolution, relatively noninvasive, and the opportunity to image a single molecule. The applications of gold nanoparticles and colloids in optical imaging and detection have been extended enormously due to their unique properties such as enhanced absorption and scattering caused by their localized surface plasmon (LSP) resonance [1]. They have a good biocompatibility where the possibility of conjugation to a variety of biomolecules and antibodies make them suitable for optical imaging and optical probes which can be used for nanoscale orientaional sensing to monitor orientations and rotations of biomolecules during their functional task [2- 4].

The influence of geometry on the optical properties of anisotropic metal nanoparticles [5-7] and the identification of their dynamics are of a great interest in such applications. A fluctuation in both scattering intensity and scattering polarization caused by the particle anisotropy is considered as a crucial point in biosensing applications and raise the necessity to study the optical anisotropy for nearly spherical nanoparticles. In this paper we assess the scope to measure the maximum anisotropy, aspect ratio, and to observe rotational dynamics for colloidal gold nanoparticles by monitoring time-traces of polarized light scattering. It has been shown that scattering by gold nanoparticles can be an alternative to fluorescence from quantum dots or dyes $[8,9]$, as they don't blink or bleach, the total scattering intensity stays constant over the entire length of the experiment, and it requires only one wavelength excitation. In polarization microscopy, rotational displacement of anisotropic particle can be observed as fluctuations in the scattering polarization, and hence, polarized light scattering is used to monitor anisotropic particle rotational dynamics [10] and to deduce its maximum anisotropy and aspect ratio. 


\section{MATERIALS AND METHODS}

Our model is a dilute suspension of gold nanoparticles suspended in water and dispersed in a viscous solvent (ethylene-glycol) with estimated mixture viscosity $\approx 0.0096 \mathrm{~Pa}$ s. This solution was deposited on a glass cover-slip. The mean diameter of the particles is $97 \mathrm{~nm}$ with a coefficient of variation $<8 \%$. As illustrated in Fig. 1, a collimated laser beam $(\lambda=532 \mathrm{~nm})$ is sent through the objective (magnification 60x, numerical aperture 0.85) toward the sample, and the same objective is used to collect the scattered light. In general, the scattered light is elliptically polarized. Polarization beam splitter splits the scattered light into two orthogonal, linearly- polarized beams such that $p$-polarized light and $s$-polarized light are detected by two photo-diodes working as polarization-balanced channels with the same intensities $I_{P}=I_{S}$. A pinhole was utilized to reduce the out-of-focus light, thus, increasing SNR.

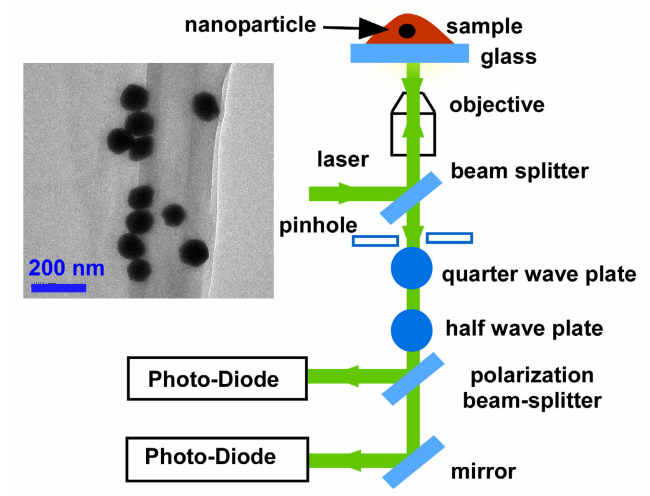

Fig. 1. Schematic diagram of the measurement setup. Inset shows TEM image of gold nanoparticles.

\section{RESULTS AND DISCUSSION}

\subsection{Experimental results}

When a particle comes into the observation volume, intensity fluctuations are observed in both orthogonal channels. In the case of isotropic particle, the difference that is detected between both channels is minimal while it becomes noticeable when the particle has some sort of anisotropy as depicted in Figs. 2(a) and (b) that shows time-traces of the two orthogonal scattering intensities from a particle, while Fig. 2(c) shows the measured anisotropy $K_{\exp }$ which is defined as:

$$
K_{\exp }=\left(I_{P}-I_{S}\right) /\left(I_{P}+I_{S}\right) \cdot
$$

In an anisotropic particle, it is the orientation of the particle relative to the excitation light polarization that causes the scattering polarization to be changed. However, when the excitation light polarization becomes parallel to either the longer or shorter axes of the particle, the scattered light polarization will not be changed. This is also similar to the case of isotropic particle, where the homogeneous morphology of the particle doesn't cause the scattering polarization to be changed and the fluctuations in the measured anisotropy-trace will be minimal. Recently, a new experimental methods and results investigating the Brownian motion of isolated anisotropic particles have been reported [11, 12]. Due to Brownian motion, particles possess both translational and rotational displacements. The 
fluctuations in the anisotropy trace as illustrated in Fig. 2(c) originate from the rotational displacement of the particle in the observation volume

In order to understand the dynamics of particles in our model, we estimated both rotational and translational diffusions. For simplicity, we approximated the particle as a prolate spheroid. The average translational diffusion coefficients for a prolate with an aspect ratio range of 1 to 1.3 is $D_{T}=4.7 \times 10^{-13}$ to $5.6 \times 10^{-13} \mathrm{~m}^{2} / \mathrm{s}$, and the average rotational diffusion coefficient is $D_{R}=136$ to $198 \mathrm{rad}^{2} / \mathrm{s}$. Calculations were done based on equations available from [13]. The calculated transition diffusion time $\tau=\omega^{2} /\left(4 D_{T}\right)$ is 65.5 to $78.3 \mathrm{~ms}$, where $\omega$ is the radius of the focal volume which was approximated to $382 \mathrm{~nm}$. This is the time that the particle spends in the observation volume. Our measurements show a very good agreement with this value as depicted in Figs. 2(a) and (b) where the measured transition diffusion time is $\sim 70 \mathrm{~ms}$. We estimated the rotational displacement $X_{R}=\left(2 D_{R} T\right)^{1 / 2}$ for an average observation time of $65.5 \mathrm{~ms}$ to $78.3 \mathrm{~ms}$ and aspect ratio range of 1 to 1.3 to be 4.2 to $5.6 \mathrm{rad}$. Even this observation time and rotational displacements were enough to detect the maximum value of the particle's anisotropy during its rotation; we believe that longer observation time is needed for more precise measurements.
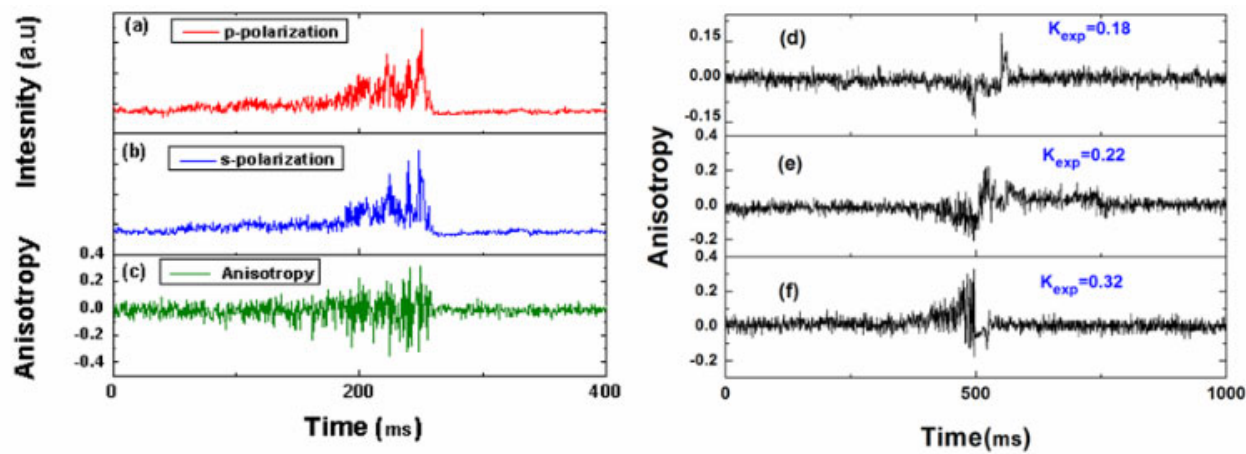

Fig. 2. (a) and (b) are time traces of two orthogonal scattering intensities $I_{P}$ and $I_{S}$ from a single gold nanoparticle. (c) Measured anisotropy of the particle extracted based on Eq. (1). (d), (e), and (f) are anisotropy time-traces for different particles. The measured maximum anisotropy values are shown. Each particle has its own anisotropy response due to the relative changes in morphologies.

When a particle reaches the observation volume, the measured intensities on both channels increase. In the case of isotropic (i.e. spherical) particle, the lateral diffusion causes correlated intensity fluctuations on both channels, while there is no effect of the rotational diffusion due to the fact that homogeneous morphology of the particle doesn't change the polarization of the excitation light. Hence, the measured anisotropy is close to zero. If the particle was anisotropic, then fluctuations in both signals are due to different types of diffusions which are: the lateral diffusion, the rotational diffusion that causes correlated intensity fluctuations and the rotational diffusion that causes anti-correlated intensity fluctuations.

It was difficult to extract rotational signals from correlation analysis because of the limited time resolution of our optical measurements. However, rotational dynamics of the particle can be observed from the anisotropy traces as shown in Fig.2 (c) and Figs. 2(d)-(f). These traces were calculated based on Eq. (1) where the lateral component of the diffusion is eliminated by dividing the difference between both intensity signals by the sum of them and thus we can observe a pure rotational motion of the particle. 


\subsection{Analytical method}

An analytical method was introduced to describe the experimental results as depicted in Fig. 3(a).
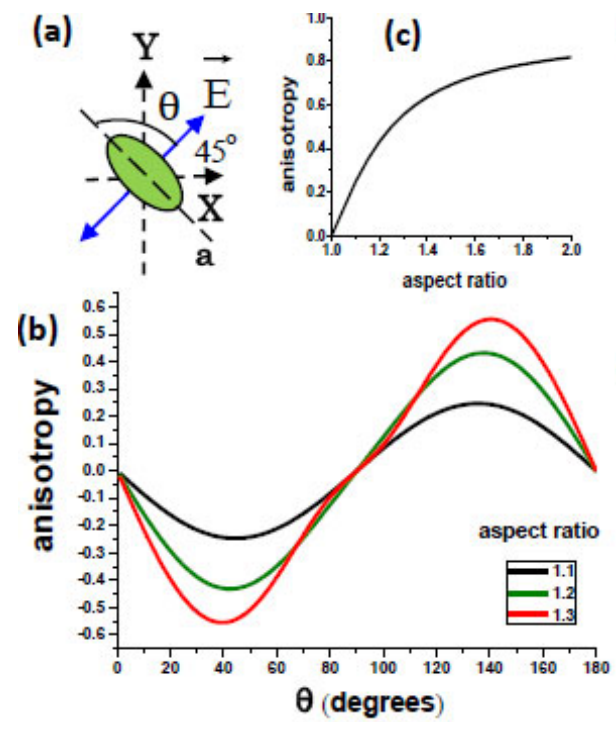

(d)

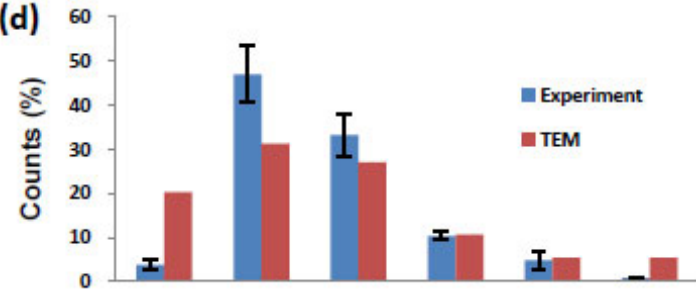

(e)
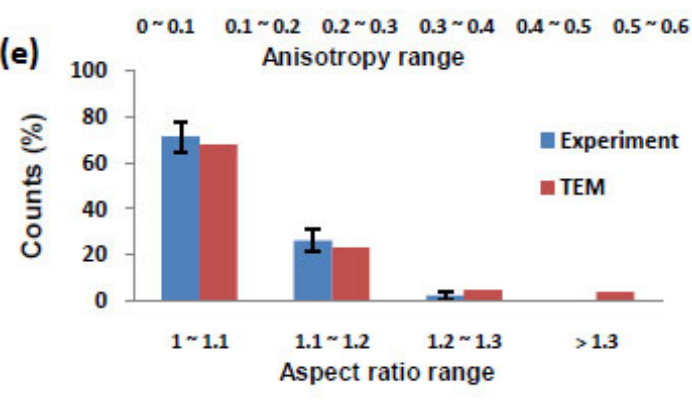

Fig. 3. (a) Schematic description of the analytical method. Here, $\theta$ is the angle between the longer axis of the particle and the $45^{\circ}$-linearly polarized light with electric field amplitude $E_{o}$. (b) Anisotropy as a function of $\theta$ for different aspect ratios calculated using Eqs. (2)-(7). The longer axis is $2 a=110 \mathrm{~nm}$, the complex index of refraction of gold at $\lambda=532 \mathrm{~nm}$ was interpolated from values available in the literature [16] to be $(0.44+i 2.43)$ and the refractive index of the mixture was approximately 1.42 . (c) Maximum value of anisotropy as a function of aspect ratio. (d) Histogram of anisotropy obtained from optical and TEM measurements (e) Histogram of aspect ratio obtained from optical and TEM measurements. Error bars are indicated.

As previously mentioned, the particle was modeled as a prolate spheroid where the induced dipole moments along the $\mathrm{X}$ and $\mathrm{Y}$ axes can be written as:

$$
\begin{aligned}
& P_{X}=\alpha_{L} E_{o} \cos \theta \cos (\theta+\pi / 4)+\alpha_{S} E_{o} \sin \theta \cos (\pi / 4-\theta), \\
& P_{Y}=\alpha_{L} E_{o} \cos \theta \sin (\theta+\pi / 4)-\alpha_{S} E_{o} \sin \theta \sin (\pi / 4-\theta),
\end{aligned}
$$

where $\alpha_{L}$ and $\alpha_{S}$ are the effective polarizabilies for major and minor axes, respectively [14], such that:

$$
\alpha_{L}=\frac{\alpha_{i}}{1-\frac{2}{3} i k^{3} \alpha_{i}-\frac{k^{2}}{2 a} \alpha_{i}}
$$




$$
\alpha_{S}=\frac{\alpha_{i}}{1-\frac{2}{3} i k^{3} \alpha_{i}-\frac{k^{2}}{2 b} \alpha_{i}},
$$

where $\alpha_{i}$ is the electrostatic polarizability, $k$ is the wave number, $a$ and $b$ are the major and minor semi-axes, respectively. This formula is a correction to the electrostatic polarizability since gold nanoparticles of diameter $50 \mathrm{~nm}$ to $100 \mathrm{~nm}$ are in the regime where the dynamical effects become important, so it is important to take into account the radiation damping and the dynamic depolarization. The electrostatic polarizability defined as [15]:

$$
\alpha_{i}=\frac{4 \pi a b^{2}}{3} \frac{\varepsilon_{p}(\omega)-\varepsilon_{m}}{\left[\left(\varepsilon_{p}(\omega)-\varepsilon_{m}\right) L_{i}+\varepsilon_{m}\right]} .
$$

Here, $\varepsilon_{p}(\omega)$ and $\varepsilon_{m}$ are the particle and medium dielectric constants, respectively, and $L_{i}$ is a geometrical factor. Based on our previous definition of anisotropy, calculated anisotropy can be defined as:

$$
K_{c a l}=\left(\left|P_{X}\right|^{2}-\left|P_{Y}\right|^{2}\right) /\left(\left|P_{X}\right|^{2}+\left|P_{Y}\right|^{2}\right) .
$$

According to our analytical method, if the particle was isotropic then $\alpha_{L}=\alpha_{S}$ and $P_{X}=P_{Y}$, hence; the detected orthogonal signals are similar and the anisotropy becomes $\sim 0$. However, if the particle was elongated then the anisotropy will take a value between 0 and 1 . By using Eqs. (2)-(7) we calculated the anisotropy as a function of $\theta$ for different aspect ratios as shown in Fig. 3 (b). Based on these graphs, the maximum value of anisotropy as a function of aspect ratio was calculated and illustrated in Fig. 3(c). By comparing the measured values of the maximum anisotropy for each particle, with those values calculated above, we can deduce the particle's aspect ratio.

A total of 100 particles were measured and the maximum values of anisotropy were found to range from 0.1 to 0.5 . A comparison between the measured distributions of maximum anisotropy and aspect ratio with those distributions calculated based on the TEM images analysis are found to show an excellent agreement confirming the validity of our approach as shown in Figs. 3(d) and (e). As it is shown, more than $70 \%$ of the particles are found to have aspect ratio of 1.1 or less and $30 \%$ are found to have aspect ratio of 1.1 to 1.3 , which reveals that the particles shape is predominately nearly spherical.

In conclusion, we presented experimental measurements for optical anisotropy and aspect ratio of colloidal gold nanoparticles using polarized light scattering. Due to the rotational diffusion of the particle in the observation volume, particle's aspect ratio could be inferred from the maximum value of the anisotropy time-trace. The high contrast, photochemical stability and high biocompatibility make polarized scattering as an alternative to fluorescence and can be utilized in an ultrasensitive molecular sensing applications such as bioconjugated nanoparticle probes. The demonstration of an optical characterization of a nanoparticle with a far-field approach opens up many perspectives for identification and quantitative study of nanoparticle via its optical signature.

\section{Acknowledgments}

This work was supported in part by a Grant-in-Aid from the Global Center of Excellence for High-Level Global Coorporation for Leading-Edge Platform on Access Spaces and a Grant- 
in-Aid for Scientific Research (S) from the Ministry of Education, Culture, Sports, Science and Technology of Japan.

\section{References}

[1] W. L. Barnes, A. Dereux, and T. W Ebbesen, "Surface plasmon subwavelength optics," Nature 424, 824-830 (2003) [doi: 10.1038/nature01937].

[2] C. Sonnichsen and A. P. Alivisatos, "Gold nanorods as novel nonbleaching plasmonbased orientation sensors for polarized single-particle microscopy," Nano Lett. 5(2), 301-304 (2005) [doi: 10.1021/nl048089k].

[3] B. Al-Qadi and T. Saiki, "A study of optical anisotropy for nearly spherical gold nanoparticles: toward a sensitive detection using polarization microscopy in biomolecular applications," Proc. SPIE 7190, $719016 \quad$ (2009) [doi:10.1117/12.808913].

[4] H. E. Grecco and O. E. Martinez, "Distance and orientation measurements in the nanometric scale based on polarization anisotropy of metallic dimers," Opt. Exp. 14(19), 8716-8721 (2006) [doi:10.1364/OE.14.008716].

[5] C. Noguez, "Surface plasmons on metal nanoparticles: the influence of shape and physical environment," J. Phys. Chem. C 111, 3806-3819 (2007) [doi:10. 1021/jp066539m].

[6] T. Kalkbrenner, U. Hakanson, and V. Sandoghdar, "Tomographic plasmon spectroscopy of a single gold nanoparticle," Nano Lett. 4, 2309-2314 (2004) [doi: $10.1021 / \mathrm{n} 1048694 \mathrm{n}]$

[7] T. Zuchner, A. V. Failla, A. Hartschuh, and A. J. Meixner, "A novel approach to detect and characterize the scattering patterns of single Au nanoparticles using confocal microscopy," J. Microsc. 229, 337-343 (2008) [doi: 10.1111/j.13652818.2008.01910.x].

[8] A. C. Zhang, T. Byassee, R. A. Tripp, and S. Nie, "Counting single native biomolecules and intact viruses with color-coded nanoparticles," Anal. Chem. 78(4), 1061-1070 (2006) [doi: 10.1021/ac051801t].

[9] C. Sonnichsen, B. M. Reinhard, J. Liphardt, and A. P. Alivisatos, "A molecular ruler based on plasmon coupling of single gold and silver nanoparticles," Nature Biotechnol. 23, 741-745 (2005) [doi: 10.1038/nbt1100].

[10] K. Drozdowicz-Tomisa, F. Xie, N. Calander, I. Gryczynski, K. Gryczynski, and E. M. Goldys, "Depolarized light scattering from colloidal gold nanoparticles," Chem. Phys. Lett. 468, 69-74 (2009) [doi:10.1016/j.cplett.2008.11.082].

[11] D. Mukhija and M. J. Solomon, "Translational and rotational dynamics of colloidal rods by direct visualization with confocal microscopy," J. Colloid Interface Sci. 314, 98-106 (2007) [doi:10.1016/j.jcis.2007.05.055].

[12] I. Hong, S. M. Anthony, and S. Granick, "Rotation in suspension of a rod-shaped colloid," Langmuir 22, 7128-7131 (2006) [doi: 10.1021/la061169e].

[13] H. Brenner, "Rheology of a dilute suspension of axisymmetric Brownian particles," Int. J. Multiphase Flow 1, 195-341 (1974) [doi:10.1016/0301-9322(74)90018-4].

[14] L. Gunnarson, T. Rindzevicius, J. Prikulis, B. Kasemo, M. Kall, S. Zou, and C. G. Schatz, "Confined plasmons in nanofabricated single silver particle pairs: Experimental observations of strong interparticle interactions," J. Phys. Chem. B 109, 1079-1087 (2005) [doi: 10.1021/jp049084e].

[15] C. F. Bohren and D. R. Huffman, Absorption and Scattering of Light by Small Particles, Wiley, New York (1983) [doi: 10.1080/716099663].

[16] P. B. Johnson and R. W. Christy, "Optical constants of the noble metals," Phys. Rev. Lett. 11(541), 4370-4379 (1969) [doi:10.1103/PhysRevB.6.4370]. 